\section{PTH-046 BENCHMARKING ENDOSCOPY SERVICES IN IRAQ - RESULTS OF A NATIONAL SURVEY}

${ }^{1,2} \mathrm{~N}$ Hawkes, ${ }^{2} \mathrm{~L}$ Al-Rubaiy*, ${ }^{3} \mathrm{~B}$ Hawkes, ${ }^{4} \mathrm{M}$ Aziz. ${ }^{1}$ Gastroenterology, Cwm Taf UHB, Llantrisant, UK; ${ }^{2}$ Welsh Endoscopy Training Network, WIMAT, Cardiff, UK; ${ }^{3}$ Endoscopy, Hywel Dda HB, Carmarthen, UK; ${ }^{4}$ Anaesthetics, Cwm Taf UHB, Llantrissant, UK

\subsection{6/gutjnl-2014-307263.492}

Introduction In the UK the Global Rating Scale (GRS) has been adopted as QA tool to improve the standards of endoscopic practice and of the patient experience. Endoscopy in Iraq is provided by a number of training and regional centres but without the level of integration seen in the NHS. We sought to benchmark practice against UK quality standards by surveying the main training centres and service providers of endoscopy in Iraq. Methods A Survey Monkey questionnaire with 40 questions relating to local endoscopic practice and based on defined areas of the GRS was sent to departmental leads in all regional centres in Iraq performing GI endoscopy by the President of the Iraqi Medical Society International. 24/35 responses (69\%) were received (all 12 major institutions responded).

Results $67 \%$ of respondents were from University Teaching Hospitals, others worked in Regional Public or Private Hospitals. Population served ranged from 100,000 to 10 million; reflected in lists performed per week (range 3 to 20+). All Units perform diagnostic upper and lower GI endoscopy. Whilst $90 \%$ perform some ERCP, only half perform $>250$ per year. Figures for EUS were similar ( $85 \%$ some EUS, $55 \%>250$ cases per year). Enteroscopy is only performed in small numbers. No agreed performance standards exist on a national level.

Access to modern endoscopes, accessories and diathermy was acceptable. Survey data aligned to the patient experience, quality of procedure, workforce and training highlighted resource and training gaps: only $70 \%$ of respondents use a structured referral form with stratification of urgent cases, $54 \%$ are able to vet appropriateness of referral and $20 \%$ can effectively audit referral practice. Written information about procedures is limited and the practice of informed consent falls short of UK standards. Numbers of recovery beds and staffing levels varied widely. Patient monitoring equipment was not universally available. $47 \%$ have an ERS, $47 \%$ paper-based records and $16 \%$ no reporting system. Morbidity and mortality, sedation practice and patient experience were recorded in less than half of responding institutions. Centres with a large numbers of trainees tend to have experienced trainers but assessment tools and training goals varied across institutions. Data on workforce was inconsistent, with conflicting reports from respondents working in the same institution.

Conclusion Web-based surveys provide a means of investigating and benchmarking endoscopic practice, via non-UK national societies, against the quality standards integral to the GRS. Resource and training gaps have been identified using this method and will inform a planned BSG sponsored visit to Iraq to deliver targeted training on quality assurance, safety and training for endoscopy. Disclosure of Interest None Declared.

\section{PTH-047 EVALUATION OF PATIENT SATISFACTION FOLLOWING PERCUTANEOUS ENDOSCOPIC GASTROSTOMY (PEG) INSERTION: DAY CASE VERSUS IN-PATIENT MODEL IN UHCW NHS TRUST}

LM Ridings*, JM Colby, NE Burch. Digestive Diseases Department, University Hospitals Coventry and Warwickshire NHS TRUST, Coventry, UK

10.1136/gutjnl-2014-307263.493
Introduction In patients with head and neck (HandN) cancer, standard practice is to insert a prophylactic gastrostomy tube to optimise nutrition and enable nutrition support during treatment. Although traditionally an inpatient procedure, many are now treated as outpatients, allowing a more time and cost effective service. There is very little data however regarding patient satisfaction with this move to a day case model.

Aim Having recently introduced a new day case PEG service for HandN cancer patients in UHCW NHS Trust, we wanted to evaluate the service and compare patient satisfaction levels in both in-patient and day case cohorts.

Methods We selected 20 sequential HandN patients who had undergone a day case PEG procedure since the day case service was introduced in March 2013. For comparison, we identified a further 20 sequential HandN patients who had undergone PEG insertion as an in-patient during the previous 12-months (Oct 2012 to Sep 2013). Deceased patients were excluded. A modified GHAA-9 questionnaire was used to assess patient satisfaction with the procedure ${ }^{[1]}$. This questionnaire was sent out retrospectively, and a pre-paid reply envelope was included with the questionnaire. Patients not responding within 1-month were telephoned to ask if they wished to complete the feedback survey.

Results Day case patients $(\mathrm{n}=20)$ were aged $40-70$ yrs (mean 54); $80 \%$ male. In-patients $(\mathrm{n}=20)$ were aged $42-81$ yrs (mean $60) ; 59 \%$ male. $75 \%$ of the day case PEGs were inserted prior to cancer treatment start, versus $45 \%$ of in-patient procedures. Those undergoing in-patient insertions utilised 53 bed-days collectively. No patient from either cohort was admitted within 7 or 30 days. There were no major complications in either group.

Patient satisfaction questionnaires were returned by 26 (65\%): 11 in-patients (55\%) and 15 day case (75\%). Mean satisfaction score for day case was $36.3 \pm 3.8$, whereas mean score for in-patients was $32.7 \pm 7.8(\max$ score $=40)$. Only $1.7 \%$ day case patients identified aspects of their PEG procedure that were fair/poor, compared to $7.9 \%$ in-patients. In-patients described higher dissatisfaction relating to time from referral to insertion, and delays waiting for insertion once admitted.

Conclusion Our results suggest greater overall satisfaction in patients undergoing PEG insertion as a day case, with no increase in complications. Moreover the PEG was undertaken in a more timely fashion with the majority $(75 \%)$ having their PEG inserted prior to treatment start. 53 patient bed days were saved for just 20 procedures which represent a cost saving to the Trust of approximately $£ 13,992^{[2]}$.

\section{REFERENCES}

1 Harewood et al. Am J Gast 2003;98:1016-1021

2 www.gov.uk/government/uploads/system/uploads/attachment_data/file/213060/ 2011-12-reference-costs-publication.pdf

Disclosure of Interest None Declared.

\section{PTH-048 PERCEPTION OF ASSESSING THE NUTRITIONAL STATUS OF INTENSIVE CARE PATIENTS: A CROSS SECTIONAL STUDY OF JORDANIAN ICU NURSES}

MT Al Kalaldeh*. Faculty of Nursing, Zarqa University, Amman, Jordan

\subsection{6/gutjnl-2014-307263.494}

Introduction Enteral nutrition is a pivotal strategy for nutrition in ICUs (Fulbrook et al. 2007). Nurses are keys to assess patients' nutritional status, detect feeding-intolerance, and curtail the prospect of complications (Persenius et al., 2006; Bourgault et al., 2007). 